\title{
Efecto del ejercicio pre-prandial sobre el metabolismo intermediario basal y post-prandial en personas con diabetes tipo 2 a grandes alturas Effect of preprandial exercise on basal and postprandial intermediary metabolism on people with type 2 diabetes at high altitudes
}

\author{
Fausto Garmendia Lorena ${ }^{1, a}$, Rosa Pando Álvarez ${ }^{1,2, b}$, William Torres Damas ${ }^{2, c}$, Wuili Valqui Cáceres ${ }^{2, d}$, Yanet Mendoza ${ }^{3, e, f}$ \\ ${ }^{1}$ Instituto de Investigaciones Clínicas, Facultad de Medicina, Universidad Nacional Mayor de San Marcos. Lima, Perú. \\ ${ }^{2}$ Hospital Nacional Dos de Mayo. Lima, Perú. \\ ${ }^{3}$ Facultad de Medicina, Universidad Nacional San Antonio Abad de Cusco. Cusco, Perú. \\ a Médico cirujano, doctor en medicina. ORCID: https://orcid.org/0000-0002-6513-8743 bMédico endocrinólogo, doctor en medicina. ORCID: https://orcid.org/0000-0002-6119-802X \\ 'Médico internista, ORCID: https://orcid.org/0000-0003-3379-2211 \\ ${ }^{\mathrm{d}}$ Médico internista, ORCID: https://orcid.org/0000-0001-7817-4252 \\ ${ }^{\text {E}}$ Licenciada en biología, doctora en ciencas de la salud pública. ORCID: https://orcid.org/0000-0003-4508-5303
}

An Fac med. 2019;80(4):465-9 / DOI: https://doi.org/10.15381/anales.v80i4.17252

\section{Correspondencia: \\ Fausto Garmendia Lorena \\ garmendiafausto@gmail.com}

Recibido: 16 de julio 2019

Aceptado:20 de setiembre 2019

Publicación en línea: 28 de diciembre 2019

Conflictos de interés: Los autores declaran no tener conflictos de interés.

Fuente de financiamiento: FEDU, Universidad Nacional Mayor de San Marcos.

Citar como: Garmendia F, Pando R, Torres W, Valqui W, Mendoza Y. Efecto del ejercicio pre-prandial sobre el metabolismo intermediario basal y post-prandial en personas con diabetes tipo 2 a grandes alturas. An Fac med. 2019;80(4):465-9. DOI: https://doi. org/10.15381/anales.v80i4.17252

\section{Resumen}

Introducción. El poblador normal de altura tiene un metabolismo intermediario diferente al del poblador de nivel del mar, caracterizado por una menor glicemia. En pacientes con diabetes tipo 2, que residen en grandes altitudes, el efecto que tiene el ejercicio aún no ha sido completamente estudiado. Objetivos. Estudiar el efecto del ejercicio, de corta duración y casual, sobre el control metabólico basal y post-prandial en personas con diabetes tipo 2 (DM2), residentes a $3395 \mathrm{msnm}$. Métodos. A 14 mujeres y 11 varones con DM2, residentes de altura (Cusco, $3395 \mathrm{msnm}$ ), de 40 a 72 años, se les efectuó dos pruebas: basal (A) y postejercicio pre-prandial (B) consistente en una caminata, durante 30 minutos, a 4 km/hora (3 METS). En ayunas y luego de un desayuno con $730 \mathrm{Kcal}(55,4 \%$ de grasas, $37,2 \%$ de carbohidratos y $7,3 \%$ de proteínas), se midió en sangre glucosa, colesterol total, HDL, triglicéridos, insulina y ácidos grasos no esterificados (AGNE) durante seis horas. Adicionalmente, se midieron niveles de colesterol VLDL, LDL, NoHDL y HOMA-IR. Resultados. Se encontró disminución estadísticamente significativa $(p<0,01)$ entre los promedios de glicemia basal $(230,2 \mathrm{mg} / \mathrm{dL})$ y post-prandial $(115 \mathrm{mg} / \mathrm{dL})$. Conclusión. El ejercicio mejoró el control glicémico postprandial en DM2 en la altura.

Palabras clave: Altitud; Diabetes Mellitus tipo 2; Ejercicio; Metabolismo (fuente: DeCS BIREME).

\section{Abstract}

Introduction. The population living on high altitudes areas has a different intermediate metabolism than people who is living on coastal areas, characterized by lower glycemia levels. In patients with type 2 diabetes, who reside at high altitudes, the effect of exercise has not yet been fully studied. Objective. To study the effect of short duration and casual exercise, on basal and post-prandial metabolic control in people with type 2 diabetes (DM2), residents at $3395 \mathrm{msnm}$. Methods. 14 women and 11 men with DM2, residents at high altitudes (Cusco, $3395 \mathrm{msnm}$ ), between 40 to 72 years old, who were intervened with two tests: baseline (A) and pre-postprandial exercise (B) consisting of a walk, for 30 minutes, at $4 \mathrm{Km} / \mathrm{h}$ (3 METS). Glucose, total cholesterol, $\mathrm{HDL}$, triglycerides, insulin and non-esterified fatty acids (AGNE) were measured in the blood on fasting and after a breakfast with $730 \mathrm{Kcal}(55,4 \%$ fat, 37,2\% carbohydrates and 7,3\% protein), for six hours. Additionally, total cholesterol, VLDL, LDL, NoHDL and HOMA-IR levels were calculated. Results. Statistically significant decrease $(p<0,01)$ was found between the mean baseline $(230,2 \mathrm{mg} / \mathrm{dL})$ and post-prandial $(115$ $\mathrm{mg} / \mathrm{dL}$ ) glycemia. Conclusion. The exercise improved post-prandial glycemic control in DM2 in people who are living in high altitudes.

Keywords: Altitude; Diabetes Mellitus, Type 2; Physical Activity; Metabolism (source: MeSH NLM). 


\section{INTRODUCCIÓN}

De acuerdo a información del censo del año 2017, la población de la sierra del Perú constituye el $28,1 \%$ de la población nacional ${ }^{(1)}$, quienes residen en un ambiente de menor presión barométrica y menor presión parcial de oxígeno que las personas que viven a nivel del mar, lo que ocasiona cambios biológicos de aclimatación a esa situación ambiental.

El poblador normal de altura (PNA) tiene un metabolismo intermediario diferente al del poblador de nivel del mar (PNM), caracterizado por una menor glicemia ${ }^{(2,3,4)}$ y concentraciones mayores de triglicéridos (Tg) y ácidos grasos no esterificados (AGNE), tanto en ayunas como en el período post-prandial ${ }^{(5)}$. Este fenómeno se da sin relación a un mayor hematocrito ni a una mayor secreción de insulina ${ }^{(6)}$; sino más bien debido a un mayor transporte y utilización de glucosa ${ }^{(7,8)}$ y a una desviación preferente del metabolismo energético hacia los lípidos, a su vez vinculada a la secreción mayor de hormonas hiperglicemientes como la somatotropina y glucagón ${ }^{(6,9)}$.

El estudio PERUDIAB ya ha descrito que en las regiones de grandes alturas existe una menor prevalencia de diabetes tipo 2 (DM2) que a nivel del mar (8,2\% en la costa y $4,5 \%$ en la altura) ${ }^{(10)}$. Adicionalmente, otros estudios han mostrado que las características clínicas en personas con DM2 ${ }^{(11)}$ y la prevalencia de diabetes tipo $1^{(12)}$, exhiben diferencias entre los residentes a nivel del mar y a grandes alturas.

Por otro lado, el régimen alimenticio y la actividad física son los pilares del tratamiento de la diabetes tipo $2^{(13,14)}$. El régimen alimenticio está orientado a mantener una buena nutrición, que permita alcanzar una glicemia normal y estable; mientras que la actividad física ocasiona, por una vía independiente a la insulina, una mayor eficiencia de los receptores de insulina y activación de la movilización de las proteínas trasportadoras de glucosa (GLUT 4), lo que produce un efecto beneficioso significativo en el control metabólico de la DM2 ${ }^{(8)}$. En el caso de la actividad física, este efecto beneficioso se ha corroborado al margen de cualquiera de los tipos que se emplee: ejercicios de naturaleza aeróbica, anaeróbica, casual o aguda, sostenida, de estiramiento, mixta; con resultados ciertamente de grado variable ${ }^{(15,16)}$.

Por las consideraciones anteriores, se justifica estudiar el efecto del ejercicio sobre el metabolismo intermediario basal y post-prandial en personas con DM2 residentes en altura, precisamente porque tienen un metabolismo diferente al de personas con diabetes tipo 2 de nivel del mar, en este caso mediante la realización de un ejercicio aeróbico, controlado, de tipo casual, pre-prandial, que se acerca al desempeño cotidiano de las personas diabéticas y no diabéticas; y en base a la hipótesis que, al igual en individuos con DM2 de nivel del mar, produciría una mejora en el control metabólico(16).

\section{MÉTODOS}

\section{Diseño del estudio}

Se realizó un estudio experimental en un grupo de 25 personas con DM2, residentes en la ciudad de Cusco (3395 msnm, presión barométrica de $510 \mathrm{mmHg}$ y presión parcial de $\mathrm{O}_{2}$ de 106,7 mmHg), 14 mujeres y 11 varones, con edades entre los 40 a 72 años, atendidos en el Laboratorio de la Facultad de Medicina de la Universidad Nacional San Antonio Abad del Cusco.

Se seleccionaron a los participantes del estudio de acuerdo a un muestreo no aleatorio por conveniencia, considerando los siguientes criterios de inclusión: 1) Tiempo de enfermedad no mayor de cinco años; 2) Sin manifestaciones clínicas de oftalmopatía, neuropatía, nefropatía ni alteraciones cardiovasculares de la DM2, debido a que dichas manifestaciones aparecen después de 5 años de enfermedad (17); y 3) Sin requerimiento terapéutico de insulina. Cuatro pacientes se encontraban con régimen alimenticio como tratamiento único; catorce recibían además glibenclamida; tres, metformina; y siete, la combinación de metformina + glibenclamida. Los hipoglicemiantes orales fueron suspendidos tres días antes de cada prueba.

\section{Intervención de estudio}

Las personas incluidas en el estudio fueron primero registradas mediante una ficha clínica, en la que se consigna información respecto a su filiación, historia actual, antecedentes personales fisiológicos, patológicos y antecedentes familiares.

Los pacientes fueron sometidos a dos pruebas: basal $(A)$ y post-ejercicio (B), en ambas se midió la glicemia en ayunas, sin medicación alguna. Por otro lado, en el período post-prandial se midieron los siguientes marcadores del metabolismo intermediario: glucosa (G), triglicéridos (Tg) por métodos enzimáticos convencionales; insulina (I) por radioinmunoensayo y AGNE por el método de Duncombe durante 6 horas ${ }^{(18)}$. El colesterol VLDL, LDL, NoHDL y el índice de resistencia a la insulina (HOMA-IR) fueron calculados por métodos estandarizados ${ }^{(19,20)}$.

La sobrecarga alimenticia consistió en un desayuno similar al de la vida cotidiana (730 Kcal) que contenía 64 g de grasas $(55,4 \%), 68 \mathrm{~g}$ de hidratos de carbono $(37,2 \%)$ y 13,4 g de proteínas (7,3\%). La actividad física consistió en una caminata durante 30 minutos a una velocidad de $4 \mathrm{Km} / \mathrm{h}$ en una faja sin fin equivalente a 3 METs. Entre ambas pruebas hubo una diferencia no mayor de dos semanas, en las cuales el mismo paciente fue su propio control.

\section{Análisis estadístico}

Se utilizó el programa IBM SPSS Statistics for Windows, Version 23.0 (IBM Corp. Released $2015{ }^{\circledR}$ ) para el cálculo de la diferencia de los promedios de las variables cuantitativas referidas a las pruebas de laboratorio solicitadas, mediante la prueba t de Student para muestras independientes y dependientes.

El efecto del ejercicio sobre el metabolismo post-prandial se midió mediante el cálculo de las diferencias de las áreas sobre la basal de los marcadores bioquímicos entre ambas pruebas, para lo cual primero se sustrae el valor de la muestra basal a cada uno de los otros valores de la prueba y el resultado final es la suma de esas diferencias.

\section{Aspectos éticos}

La investigación fue aprobada por el Comité de Ética del Hospital Nacional Dos de Mayo. Se solicitó el consentimiento informado a los participantes del estudio. 


\section{RESULTADOS}

Al evaluar las características generales de los participantes del estudio, se aprecia que la edad fue semejante, los varones tuvieron mayor peso y talla que las mujeres, el IMC fue semejante; no hubo diferencias en los marcadores bioquímicos incluyendo a la hemoglobina glicosilada (HbA1c) e índice HOMA (Tabla 1). la basal de las pruebas A y B, se encontró diferencias significativas para las concentraciones de glucosa (Figura 1); en cambio, no hubo diferencias en las áreas de TG, insulina, ni AGNE (Tabla 2).

\section{DISCUSIÓN}

La disminución significativa de las áreas sobre la basal de las concentraciones de glucosa entre las pruebas A y $B$ del período post-prandial fue el hallazgo más destacado de esta investigación; lo cual permite señalar que una actividad física casual, de corta duración y pre-prandial también tiene un efecto beneficioso sobre el control de la glicemia de las per-
En la comparación de las áreas sobre

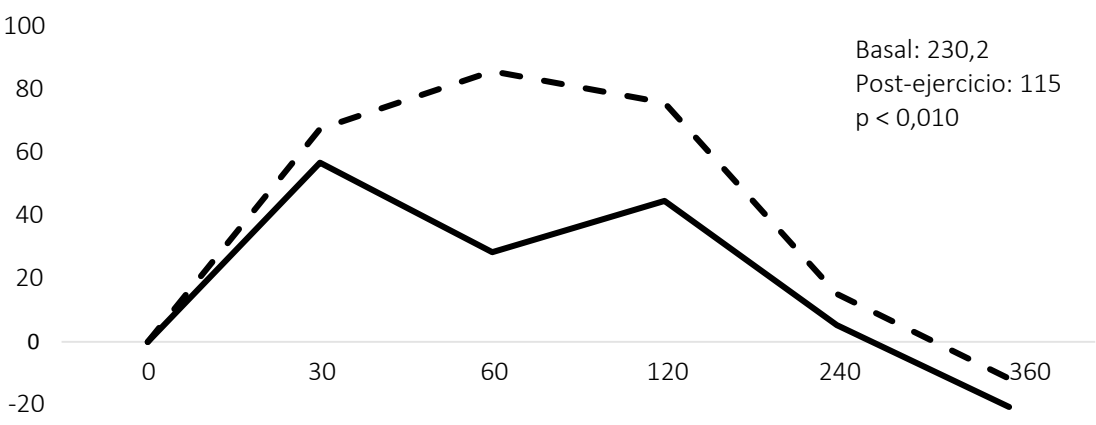

$-40$

$$
\text { - basal Post-ejercicio: }
$$

Figura 1. Comparación de las áreas sobre el basal de las concentraciones de glucosa en el periodo post-prandial, en pacientes con DM2 residentes a 3395 msnm. sonas con DM2 residentes en la altura. A diferencia de lo hallado a nivel del mar, en donde bajo las mismas condiciones metodológicas, no se encontró diferencias en las áreas de insulina y AGNE ${ }^{(16)}$.

No haber encontrado una disminución significativa de la concentración de Tg y AGNE por efecto del ejercicio podría explicarse por el hecho que el PNA tienen una respuesta metabólica durante el ejercicio diferente al del PNM ${ }^{(6)}$. Así,
Tabla 1. Características generales de pacientes con DM2, residentes en la ciudad de Cusco (3395 msnm).

\begin{tabular}{lccc} 
Características & Mujeres (n=14) & Varones $\mathbf{( n = 1 1 )}$ & Valor $\mathbf{p}$ \\
\hline Edad (años) & $56,0 \pm 9,3$ & $57,7 \pm 9,9$ & 0,66 \\
\hline Peso (Kg) & $65,8 \pm 7,9$ & $73,4 \pm 4,9$ & 0,007 \\
\hline Talla (m) & $1,53 \pm 0,07$ & $1,66 \pm 0,06$ & $<0,001$ \\
\hline IMC (Kg/m²) & $27,6 \pm 1,8$ & $26,3 \pm 1,9$ & 0,097 \\
\hline CT (mg/dL) & $222,2 \pm 45,7$ & $203,3 \pm 20,7$ & 0,184 \\
\hline HDL (mg/dL) & $39,7 \pm 11,2$ & $46,8 \pm 25,0$ & 0,398 \\
\hline Trigliceridos (mg/dL) & $278,7 \pm 188,3$ & $214,5 \pm 46,2$ & 0,238 \\
\hline VLDL (mg/dL) & $40,5 \pm 17,4$ & $39,6 \pm 10,0$ & 0,883 \\
\hline LDL (mg/dL) & $121,1 \pm 28,4$ & $116,7 \pm 31,4$ & 0,731 \\
\hline No HDL (mg/dL) & $182,5 \pm 49,4$ & $156,5 \pm 36,1$ & 0,138 \\
\hline Glucosa (mg/dL) & $137,7 \pm 55,2$ & $140,2 \pm 68,8$ & 0,923 \\
\hline Insulina (Ul/mL) & $10,2 \pm 12,7$ & $10,4 \pm 7,4$ & 0,959 \\
\hline NEFA (mMol/L) & $710,5 \pm 187,6$ & $614,0 \pm 194,4$ & 0,225 \\
\hline HbA1c (\%) & $7,95 \pm 1,98$ & $8,60 \pm 3,10$ & 0,553 \\
\hline HOMA & $3,22 \pm 3,34$ & $3,06 \pm 1,83$ & 0,874 \\
\hline
\end{tabular}

IMC: Índice de masa corporal; CT: Colesterol total; HDL: Lipoproteínas de alta densidad; VLDL: Lipoproteínas de muy baja densidad; LDL: Lipoproteínas de baja densidad; NEFA: Ácidos Grasos no esterificados; HbA1c: Hemoglobina glicosilada; HOMA: Índice de resistencia a la insulina. frente al ejercicio sub-máximo en ayunas, no se observa un incremento en la somatotropina como si lo hace el PNM. Por otro lado, el PNA tiene concentraciones mayores de Tg y AGNE que los PNM; asimismo, los pacientes con DM2 tienen cifras más altas que las personas no diabéticas, por lo que se debe tomar en cuenta el contenido de lípidos de la sobrecarga alimenticia administrada en el desayuno, las cuales incrementan la respuesta postprandial de triglicéridos ${ }^{(21)}$.

El objetivo terapéutico central de control metabólico de las personas con diabetes es alcanzar cifras normales de la glicemia, $\mathrm{HbA} 1 \mathrm{c}$ menor de 6,5\%, así como el control de la presión arterial y del perfil lipídico, con lo que se evita el desarrollo de las manifestaciones crónicas de la DM y con ello se consigue una mejor calidad de vida de los pacientes. Para conseguir ese objetivo, es indispensable que las personas con diabetes adopten un estilo de vida saludable; primero, mediante en un régimen alimenticio que les proporciones un aporte balanceado de macro y micro nutrientes y alcancen un IMC normal y, en segundo lugar, la realización de actividad física regular.

La actividad física debe estar condicionada al estado de salud del paciente, sus preferencias y facilidades. Una de las formas más prácticas, a la que se suelen amoldar la mayoría de los pacientes consiste en efectuar caminatas diarias, que pueden formar parte de las actividades cotidianas, se las puede efectuar con in- 
Tabla 2. Comparación de las áreas sobre la basal de las concentraciones de indicadores bioquímicos post-prandiales, antes y después de la intervención de estudio en pacientes con DM2 residentes a $3395 \mathrm{msnm}$

Indicador Bioquímico

Basal

Post-ejercicio

Valor $p$

\begin{tabular}{cccc} 
Insulina & & & \\
\hline 30 min & $22,6 \pm 18,5$ & $23,0 \pm 21,2$ & 0,937 \\
\hline 60 min & $37,1 \pm 32,1$ & $25,7 \pm 18,5$ & 0,133 \\
\hline 120 min & $44,5 \pm 51,3$ & $27,7 \pm 17,8$ & 0,141 \\
\hline 240 min & $12,0 \pm 13,6$ & $10,4 \pm 11,6$ & 0,666 \\
\hline 360 min & $1,5 \pm 6,3$ & $0,5 \pm 5,6$ & 0,249 \\
\hline Suma & $116,0 \pm 110,3$ & $83,8 \pm 52,3$ & 0,197 \\
\hline Triglicéridos & & & 0,152 \\
\hline 30 min & $-9,3 \pm 31,0$ & $5,3 \pm 39,8$ & 0,316 \\
\hline 60 min & $19,7 \pm 51,1$ & $36,0 \pm 62,0$ & 0,044 \\
\hline 120 min & $32,7 \pm 47,1$ & $71,4 \pm 79,7$ & 0,713 \\
\hline 240 min & $92,8 \pm 67,3$ & $85,1 \pm 76,9$ & 0,408 \\
\hline 360 min & $95,2 \pm 69,0$ & $77,0 \pm 84,5$ & 0,500 \\
\hline Suma & $229,8 \pm 182,8$ & $275,0 \pm 277,6$ & \\
\hline Ácidos grasos no & & & 0,786 \\
esterificados (AGNE) & & & 0,762 \\
\hline 30 min & $-137,5 \pm 161,7$ & $-148,4 \pm 115,6$ & 0,861 \\
\hline 60 min & $-287,3 \pm 222,1$ & $-270,0 \pm 176,3$ & 0,725 \\
\hline 120 min & $-310,2 \pm 221,7$ & $-320,5 \pm 188,7$ & \\
\hline 240 min & $-161,4 \pm 213,4$ & $-182,0 \pm 199,3$ & \\
\hline 360 min & $169,0 \pm 225,3$ & $60,3 \pm 235,8$ & $-860,8 \pm 733,9$ \\
\hline Suma & $-715,1 \pm 822,8$ & & \\
\hline
\end{tabular}

tervalos, no requiere el uso de ropa deportiva, es aeróbica, se puede realizar en cualquier hora del día, puede ser realizada por personas de todas las edades ${ }^{(22,23)}$. En mujeres mayores, se ha demostrado que caminatas entre 4000 a 7500 pasos diarios, equivalentes aproximadamente a 2,4 a 4,5 km, disminuyen la mortalidad por todas las causas, incluyendo a personas con DM2 (24)

Una limitación de nuestro estudio es no haber obtenido información de la variación de la $\mathrm{HbA} 1 \mathrm{c}$; sin embargo, el hecho que las dos pruebas se efectuaron con un lapso menor de dos semanas y que el ejercicio no duró más de 30 minutos hace poco probable que se hubiera encontrado diferencias significativas.

Se concluye, que la actividad física pre-prandial, de corta duración y casual, tiene un efecto inmediato de mejorar post-prandial de residentes en la altura con DM2, con lo que se amplía el conocimiento del efecto del ejercicio sobre el control metabólico de la DM2.

\section{REFERENCIAS BIBLIOGRÁFICAS}

1. Instituto Nacional de Estadistica e Informática. Perú: Perfil Sociodemográfico. Informe Nacional. Censos Nacionales 2017: XII de Población, VII de Vivienda y III de Comunidades Indigenas. [On line]. Instituto Nacional de Estadística e Informática; 2018.

2. Cassinelli CM. Glucosa, ácido láctico y ácido pirúvico a nivel del mar y en la altura. Anales de la Facultad de Medicina. 1949;32(1):1-28. DOI: 10.15381/anales.v32i1.9582

3. Koufakis T, Karras SN, Mustafa OG, Zebekakis P, Kotsa K. The Effects of High Altitude on Glucose Homeostasis, Metabolic Control, and Other Diabetes-Related Parameters: From Animal Studies to Real Life. High Alt Med Biol. 2019;20(1):111. DOI: 10.1089/ham.2018.0076 el control de la glicemia en el período
4. Garmendia F, Pando R, Mendoza Y, Torres W, Urdanivia E. Basal and postprandial intermediary metabolism in normal male high altitude dwellers. MOJ Biology and Medicine. 2018;3(1):46. DOI: 10.15406/mojbm.2018.03.00065

5. Garmendia F, Pando R, Mendoza Y, Torres W Metabolismo intermediario basal y posprandial en mujeres posmenopáusicas normales de altura. Revista Peruana de Ginecologia y Obstetricia. 2019;65(2):153-6. DOI: 10.31403/rpgo.v65i2164

6. Sutton JR, Garmendia F. Hormonal Responses to Exercise at Altitude in Sea Level and Mountain Man. Dans: Brendel W, Zink RA, directeurs. High Altitude Physiology and Medicine. New York, NY: Springer; 1982. p. 165-71.

7. Loike JD, Cao L, Brett J, Ogawa S, Silverstein SC, Stern D. Hypoxia induces glucose transporter expression in endothelial cells. Am J Physiol. 1992;263(2 Pt 1):C326-333. DOI: 10.1152/ ajpcell.1992.263.2.C326

8. Bashan N, Burdett E, Hundal HS, Klip A. Regulation of glucose transport and GLUT1 glucose transporter expression by $\mathrm{O} 2$ in muscle cells in culture. $\mathrm{Am} \mathrm{J}$ Physiol. 1992;262(3 Pt 1):C682-690. DOI: 10.1152/ ajpcell.1992.262.3.C682

9. Gonzales G, Coyotupa J, Guerra Garcia R. Elevated levels of growth hormone in natives from high altitude. Interrelationship with glucose levels. Acta Andina. 1992;1:85-8.

10. Seclen SN, Rosas ME, Arias AJ, Huayta E, Medina CA. Prevalence of diabetes and impaired fasting glucose in Peru: report from PERUDIAB, a national urban population-based longitudinal study. BMJ Open Diabetes Research and Care. 2015;3(1). DOI: 10.1136/bmjdrc-2015-000110

11. Garmendia F. Influencia de la altura sobre la evolución clínica y bioquímica de la diabetes mellitus. Rev Asoc Med Peruano Norteamericana. 1984;2:6-7.

12. Karvonen M, Viik-Kajander M, Moltchanova $E$, Libman I, LaPorte R, Tuomilehto J. Incidence of childhood type 1 diabetes worldwide. Diabetes Mondiale (DiaMond) Project Group. Diabetes Care. 2000;23(10):1516-26. DOI: 10.2337/diacare.23.10.1516

13. Andrews RC, Cooper AR, Montgomery AA, Norcross AJ, Peters TJ, Sharp DJ, et al. Diet or diet plus physical activity versus usual care in patients with newly diagnosed type 2 diabetes: the Early ACTID randomised controlled trial. Lancet. 2011;378(9786):129-39. DOI: 10.1016/S01406736(11)60442-X

14. American Diabetes Association. Summary of Revisions: Standards of Medical Care in Diabetes-2019. Diabetes Care. 2019;42(Supplement 1):S4-6. DOI: 10.2337/dc19-Srev01

15. Asano RY, Sales MM, Browne RAV, Moraes JFVN, Coelho Júnior HJ, Moraes MR, et al. Acute effects of physical exercise in type 2 diabetes: A review. World J Diabetes. 2014;5(5):659-65. DOI: 10.4239/ wjd.v5.i5.659

16. Garmendia F, Pando R, Torres W, Valqui W. Efecto del ejercicio preprandial sobre el metabolismo intermediario basal y postprandial en pacientes con diabetes tipo 2 de nivel del mar. Anales de la Facultad de Medicina. 2019;80(2):173-6. DOI: 10.15381/anales.802.16046 
17. Garmendia F. Manual de Endocrinologia y Metabolismo. Lima: Instituto Nacional de Salud; 2014

18. Duncombe WG. The colorimetric micro-determination of non-esterified fatty acids in plasma. Acta Clinica Chimica. 1964;9(2):122-5. DOI: 10.1016/00098981(64)90004-X

19. Friedewald WT, Levy RI, Fredrickson DS. Estimation of the concentration of low-density lipoprotein cholesterol in plasma, without use of the preparative ultracentrifuge. Clin Chem. 1972;18(6):499-502.

20. Matthews DR, Hosker JP, Rudenski AS, Naylor BA, Treacher DF, Turner RC. Homeostasis model as- sessment: insulin resistance and beta-cell function from fasting plasma glucose and insulin concentrations in man. Diabetologia. 1985;28(7):412-9. DOI: 10.1007/bf00280883

21. Dubois C, Beaumier G, Juhel C, Armand M, Portugal $\mathrm{H}$, Pauli AM, et al. Effects of graded amounts (0-50 g) of dietary fat on postprandial lipemia and lipoproteins in normolipidemic adults. Am J Clin Nutr. 1998;67(1):31-8. DOI: 10.1093/ajcn/67.1.31

22. Haxhi J, Leto G, di Palumbo AS, Sbriccoli P, Guidetti L, Fantini $C$, et al. Exercise at lunchtime: effect on glycemic control and oxidative stress in middle-aged men with type 2 diabetes. Eur J Appl Physiol. 2016;116(3):573-82 DOI: 10.1007/s00421-015-3317-3

23. Borror A, Zieff G, Battaglini C, Stoner L. The Effects of Postprandial Exercise on Glucose Control in Individuals with Type 2 Diabetes: A Systematic Review. Sports Med. 2018;48(6):1479-91. DOI: 10.1007/ s40279-018-0864-X

24. Lee I-M, Shiroma EJ, Kamada M, Bassett DR, Matthews CE, Buring JE. Association of Step Volume and Intensity With All-Cause Mortality in Older Women. JAMA Intern Med. 2019;179(8):1105-12. DOI: 10.1001/jamainternmed.2019.0899 